OPEN ACCESS

Edited by:

Dina Popovic,

Sheba Medical Center, Israel

Reviewed by:

Jules Angst,

Psychiatric University Hospital

Zurich, Switzerland

Michele Fornaro,

New York State Psychiatric Institute

(NYSPI), United States

*Correspondence:

Gang Wang

gangwangdoc@gmail.com

tThese authors have contributed equally to this work

Specialty section: This article was submitted to

Mood and Anxiety Disorders,

a section of the journal

Frontiers in Psychiatry

Received: 09 December 2019 Accepted: 20 May 2021

Published: 22 June 2021

Citation:

Wang $Y$-Y, Feng $Y$, Fang $M$, Guo $C$, Ungvari GS, Hall BJ, Wang G and

Xiang Y-T (2021) Comparing Screening Abilities of the 33-Item Hypomania Checklist (HCL-33) and the 33-Item Hypomania Checklist External Assessment (HCL-33-EA) for the Detection of Bipolar Disorder.

Front. Psychiatry 12:518722. doi: 10.3389/fpsyt.2021.518722

\section{Comparing Screening Abilities of the 33-Item Hypomania Checklist (HCL-33) and the 33-Item Hypomania Checklist External Assessment (HCL-33-EA) for the Detection of Bipolar Disorder}

\author{
Yuan-Yuan Wang ${ }^{1 \dagger}$, Yuan Feng ${ }^{2 \dagger}$, Meng Fang ${ }^{2 \dagger}$, Chengwei Guo ${ }^{2 \dagger}$, Gabor S. Ungvari ${ }^{3,4}$, \\ Brian J. Hall ${ }^{5,6}$, Gang Wang ${ }^{2 \star}$ and Yu-Tao Xiang ${ }^{7,8,9}$ \\ 1 Division of Psychology, Faculty of Health and Life Sciences, De Montfort University, Leicester, United Kingdom, \\ ${ }^{2}$ The National Clinical Research Center for Mental Disorders, Beijing Key Laboratory of Mental Disorders, Beijing Anding \\ Hospital and the Advanced Innovation Center for Human Brain Protection, Capital Medical University, Beijing, China, \\ ${ }^{3}$ University of Notre Dame Australia, Fremantle, WA, Australia, ${ }^{4}$ Division of Psychiatry, School of Medicine, University of \\ Western Australia/Graylands Hospital, Perth, WA, Australia, ${ }^{5}$ New York University Shanghai, Shanghai, China, ${ }^{6}$ School of \\ Global Public Health, New York University, New York, NY, United States, ${ }^{7}$ Unit of Psychiatry, Department of Public Health and \\ Medicinal Administration, Institute of Translational Medicine, Faculty of Health Sciences, University of Macau, Macao, China, \\ ${ }^{8}$ Centre for Cognitive and Brain Sciences, University of Macau, Macao, China, ${ }^{9}$ Institute of Advanced Studies in Humanities \\ and Social Sciences, University of Macau, Macao, China
}

Background: Bipolar disorder (BD) is a severe psychiatric disorder that is often misdiagnosed and under-diagnosed in clinical settings. The 33-item Hypomania Checklist (HCL-33) is a newly developed self-administered scale for BD detection, while the 33-item Hypomania Checklist-external assessment (HCL-33-EA) is a version of the HCL-33 for external rating used by patient's carer (e.g., family member or friend). We aimed to compare the screening abilities between the HCL-33 and the HCL-33-EA, and evaluate the screening consistency between the two scales.

Methods: The data were collected from 269 patients with diagnosed BD ( $n=84$ ) or major depressive disorder (MDD) $(n=185)$. The sensitivity, specificity, positive predictive value (PPV), negative predictive value (NPV), and area under the curve (AUC) between the HCL-33 and the HCL-33-EA for BD were compared against clinician diagnosis as the gold standard.

Results: Using Youden's index, the optimal cut-off value of the HCL-33 is 20, while the corresponding figure for HCL-33-EA is 11. Using Youden's index, the HCL-33-EA showed a better performance than the HCL-33 (0.51 vs.0.41). The HCL-33-EA was more sensitive in correctly identifying BD patients from MDD patients than the HCL-33 (0.83 vs. 0.59), while the HCL-33 presented better specificity than the HCL-33-EA (0.82 vs. 0.68). There was significant screening consistency between the two scales $(p<0.001)$. 


\begin{abstract}
Conclusions: Both scales have acceptable psychometric properties in detection BD from MDD. Use of the two scales should be considered based on the assessment purpose in clinical research or daily practice (i.e., prefer sensitivity or specificity). Noticeably, the current sample size is insufficient and future studies are recommended to further evaluate the scales.
\end{abstract}

Keywords: bipolar disorder, sensitivity, specificity, HCL-33-EA, HCL-33

\section{BACKGROUND}

Bipolar disorder (BD) is a chronic and severe mood disorder comprising depressive and manic/hypomanic episodes (1). BD is frequently under-recognized, partly because patients are misdiagnosed as having major depressive disorder (MDD) during depressive episodes of $\mathrm{BD}(1-3)$. Moreover, $\mathrm{BD}$ patients usually do not report hypomanic episodes to clinicians, since patients may not experience impairment, and therefore do not consider hypomania as BD symptoms (2). Due to the commonly neglected hypomania, it is difficult to estimate the prevalence of misdiagnosed and underdiagnosed $\mathrm{BD}$ among those treated as MDD. Studies found that an average of 10 years was needed before accurate diagnoses of $\mathrm{BD}$ were established; in addition, around one-third of $\mathrm{BD}$ patients experienced at least once misdiagnosis $(4,5)$. The misdiagnosis of $\mathrm{BD}$ may have serious consequences, including high suicide risk (6) and low antidepressant treatment efficacy (7). Thus, it is crucial to distinguish $\mathrm{BD}$ accurately from other disorders, particularly MDD.

The Hypomania Checklists (HCL) are a series of widely used scales in detecting hypomanic symptoms and identifying $\mathrm{BD}$, such as the 32-item Hypomania Checklist (HCL-32) (8), the 33-item Hypomania Checklist (HCL-33) (9), the 33-item Hypomania Checklist-external assessment (HCL-33-EA) (10), and their short versions $(11,12)$. The HCL-32 is a widely used patient-rated screening instrument for hypomanic symptoms with good psychometric properties in differentiating BD from MDD (8) and has been widely used in different countries (1318). The HCL-33 is a recently developed questionnaire based on the extension of the HCL-32, which provides a more detailed assessment of hypomanic symptoms (9). The HCL-33-EA is the external assessment version of the HCL-33, which was designed to assess hypomanic symptoms by carers (such as spouses, parents, and friends) (10).

Several studies on the screening ability of the HCL-33 and the HCL-33-EA found satisfactory BD screening abilities of the two scales $(9,19)$. The consistency between the HCL-33 and the HCL-33-EA has been evaluated in a sample of Polish adults, which showed sufficient consistency between them (10). However, comparison between the HCL-33 and the HCL-33-EA for the detection of $\mathrm{BD}$ in patients with $\mathrm{MDD}$ and $\mathrm{BD}$ has not been conducted in China. The optimal cut-off value of the HCL-33 for distinguish BD from MDD is 15 in China (9),

Abbreviations: HCL-33, 33-item Hypomania Checklist (HCL-33); HCL-33-EA, the 33-item Hypomania Checklist External Assessment. while the corresponding values of HCL-33-EA were not reported $(10,19)$. This is a critical gap in the literature, since there are an estimated 1.54 million people in China with BD (20). We aimed to compare the psychometric properties of the HCL33 and the HCL-33-EA, including scale reliability, sensitivity, specificity, positive predictive value (PPV), negative predictive value (NPV), and area under the curve (AUC). In addition, we also measured the consistency between the HCL-33 and the HCL-33-EA, and aimed to provide optimal cut-off values of the HCL-33 and HCL-33-EA for distinguishing of BD from MDD in Chinese patients.

\section{METHOD}

\section{Participants and Site}

Following previous studies on psychometric properties of the HCL scales (11, 21-23), 269 inpatients and the same number of their carers were consecutively recruited between October, 2016 and January, 2019 in a major tertiary psychiatric hospital in Beijing, China. The patients were included if they were (1) adult patients diagnosed as MDD or BD depressive episode by two psychiatrists using the Mini-International Neuropsychiatric Interview Version $5.0(24,25)$ according to the International Classification of Diseases (ICD-10) (26), which was confirmed by a review of medical records; (2) could understand the contents of the interview. Patients with MDD or BD secondary to major medical conditions were excluded. All patients and their carer provided written consent and the Ethics Committee of Beijing Anding Hospital approved the study protocol.

\section{Assessments}

Basic demographic characteristics of patients and their carers were collected. The Chinese version of the HCL-33 was used with patients, and the HCL-33-EA was used with their carers. The HCL-33 (9) and HCL-33-EA (10) are self-rated questionnaires on patients' hypomanic symptoms. The total scores of the two scales are calculated by adding up all the positive answers and the total score ranges from 0 to $33(9,10)$. The Chinese versions of the two scales have been validated previously $(9,19)$.

\section{Statistical Analyses}

The sample size was calculated using $G^{*}$ power (27). Using reported screening abilities of HCL scales $(9,12)$ and the allocation ratio of $\mathrm{MDD}$ and $\mathrm{BD}$ patients as reported previously $(4,5)$ and given the alpha error probability of 0.05 , and a conservative medium effect size of 0.5 , at least 294 participants, with $68 \mathrm{BD}$ and $226 \mathrm{MDD}$ patients, would be needed. 
TABLE 1 | Characteristics of patients with mood disorders and their carers.

\begin{tabular}{|c|c|c|c|c|c|c|}
\hline & \multirow{2}{*}{\multicolumn{2}{|c|}{ Carers $(n=269)$}} & \multicolumn{4}{|c|}{ Patients $(n=269)$} \\
\hline & & & \multicolumn{2}{|c|}{$\operatorname{MDD}(n=185)$} & \multicolumn{2}{|c|}{$\mathrm{BD}(n=84)$} \\
\hline & $N$ & $\%$ & $N$ & $\%$ & $N$ & $\%$ \\
\hline Men & 149 & 55.4 & 42 & 22.7 & 22 & 26.2 \\
\hline Married & 236 & 87.7 & 113 & 61.1 & 37 & 44.0 \\
\hline \multirow[t]{2}{*}{ Employed } & 266 & 98.9 & 164 & 88.6 & 73 & 86.9 \\
\hline & Mean & SD & Mean & SD & Mean & SD \\
\hline Age (years) & 42.7 & 11.7 & 35.9 & 12.8 & 32.9 & 12.4 \\
\hline Education (years) & 13.1 & 2.9 & 13.9 & 3.1 & 14.1 & 2.8 \\
\hline
\end{tabular}

$B D$, bipolar disorder; $M D D$, major depressive disorder.

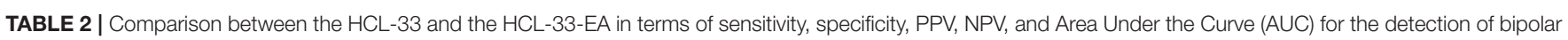
disorder from major depressive disorder.

\begin{tabular}{|c|c|c|c|c|c|c|c|c|}
\hline Scales & AUC & $95 \% \mathrm{Cl}$ & Cut-off value & Sensitivity (SE) & Specificity (SP) & Youden's J & PPV & NPV \\
\hline \multirow[t]{4}{*}{ HCL-33 } & 0.73 & $0.66-0.81$ & 18 & 0.66 & 0.71 & 0.37 & 0.52 & 0.84 \\
\hline & & & 19 & 0.62 & 0.78 & 0.39 & 0.57 & 0.83 \\
\hline & & & $20^{a}$ & 0.59 & 0.82 & 0.41 & 0.60 & 0.82 \\
\hline & & & 21 & 0.47 & 0.87 & 0.34 & 0.63 & 0.79 \\
\hline \multirow[t]{4}{*}{ HCL-33-EA } & 0.82 & $0.77-0.88$ & 9 & 0.89 & 0.55 & 0.43 & 0.45 & 0.91 \\
\hline & & & 10 & 0.86 & 0.61 & 0.46 & 0.47 & 0.89 \\
\hline & & & $11^{\mathrm{a}}$ & 0.83 & 0.68 & 0.51 & 0.50 & 0.89 \\
\hline & & & 12 & 0.75 & 0.74 & 0.49 & 0.53 & 0.86 \\
\hline
\end{tabular}

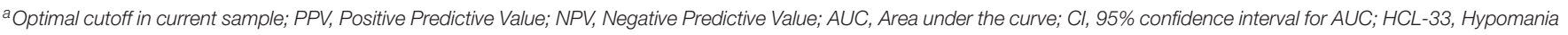
Checklist-33; HCL-33-EA, Hypomania Checklist-33-externl assessment.

SPSS 25 (IBM Corp, Armonk) was used for all analyses. The HCL-33 and the HCL-33-EA were compared by sensitivity, specificity, PPV and NPV. The Receiver Operating Characteristic (ROC) curve was plotted to represent the ability of the instrument to distinguish between $\mathrm{BD}$ and MDD. The internal consistency was measured by the Cronbach's alpha, in which excellent $\alpha$ coefficient was defined as $\geq 0.90$, good was defined as $0.80-0.89$, and adequate was defined as $0.70-0.79$ (28). Following previous studies $(12,29)$, the optimal cut-off was calculated using the maximum sum score of sensitivity + specificity -1 , according to Youden's index (30). Cohen's kappa was used to determine the consistency between HCL-33 and the HCL-33-EA, with below 0.40 as poor agreement, $0.40-0.75$ as fair to good agreement, and above 0.75 as excellent agreement (31). Statistical significance was set at was set at $<0.05$ (two-tailed).

\section{RESULTS}

A total number of 269 patients (MDD: $n=185$ and BD: $n=84$ ), and 269 carers who met the study criteria were included during the study period for analyses. Their basic characteristics are shown in Table 1.

The Cronbach's alpha of the HCL-33 was 0.867 and that the HCL-33-EA was 0.872, which suggests that the two scales had good reliability, while the HCL-33-EA had slightly higher reliability than the HCL-33. The mean sum score of the HCL-33 and the HCL-33-EA were $15.3(\mathrm{SD}=6.5)$ and $11.0(\mathrm{SD}=6.2)$, respectively. Using the optimal cut-offs calculated in the current sample, there was a significant, but poor agreement between the HCL-33 and the HCL-33-EA $(k=0.36, p<0.001)$. The proportion of $\mathrm{BD}$ was relatively higher in the current sample than the calculated proportion, thus the chance agreement should be adjusted according to the influence of prevalence and bias (32). The Prevalence and Bias Adjusted Kappa (PBAK) was also calculated $(k=0.37$, prevalence index $=-0.22$, bias index $=$ -0.17). As shown in Table 2, the HCL-33 and HCL-33-EA was compared in terms of sensitivity, specificity, Youden's J, PPV, NPV, and AUC, using the optimal cut-offs of the two scales.

Using Youden's index, the optimal cut-off value of the HCL33 is 20 , while the optimal cut-off value for HCL-33-EA is 11 . The HCL-33-EA showed a better performance than the HCL-33 in discriminating $\mathrm{BD}$ from MDD (the maximum of sensitivity + specificity -1 : 0.51 vs. 0.41 ; Table 2). Figure 1 shows the ROC curves of the HCL-33 and the HCL-33-EA. The HCL-33EA demonstrated better sensitivity than the HCL-33 (0.83 vs. 0.59 ) to correctly identify patients with $\mathrm{BD}$ from patients with MDD, while the HCL-33 presented better specificity than the HCL-33-EA (0.82 vs. 0.68). 


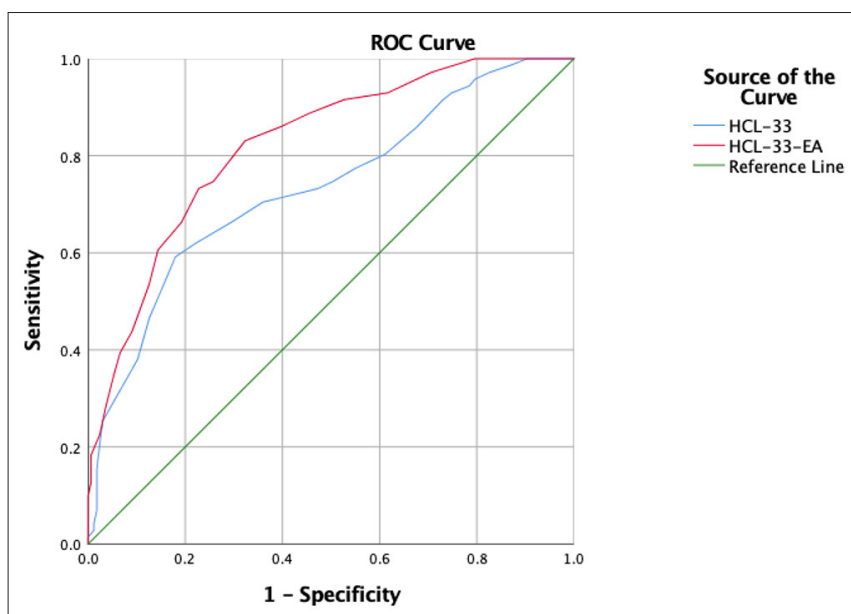

FIGURE 1 | ROC curves for the HCL-33 and the HCL-33-EA. HCL-33, Hypomania Checklist-33; HCL-33-EA, Hypomania Checklist-33-external assessment.

\section{DISCUSSION}

This was the first study to compare the screening abilities of the Chinese version of the HCL-33 and the HCL-33-EA and their consistency in identifying BD from MDD. In terms of AUC, the HCL-33-EA showed better performance than the HCL-33, although the difference did not reach significance. The HCL-33EA had higher sensitivity and the HCL-33 had higher specificity. There is no significant difference between the two scales in terms of detection $\mathrm{BD}$ from MDD, and thus we recommended the joint use of the two scales. Moreover, the relatively low Youden's $J$ suggests that positive screenings should be confirmed in formal diagnostic interviews with a mental health professional. While a high sensitivity is the key property of a screening instrument, the relatively lower specificity indicates that false positives will likely be included in positive screenings.

Consistent with previous findings (10), this study found the HCL-33-EA was useful in assessing hypomanic symptoms. Similar to other studies (19), we found that the HCL-33-EA had higher reliability and the HCL-33-EA total score was lower than the HCL-33. This is also the first study that calculated an optimal cut-off for the HCL-33-EA. For discriminating BD from MDD, the optimal cut-off for the HCL-33-EA was 11 in the Chinese population, which is lower than the optimal cut-off of 20 for the HCL-33 in this study and 15 in a previous study (9) as expected. The discrepancy in cut-off values could be due to the different reflection and observation on hypomanic symptoms between patient's and their carer's assessments.

The HCL-33-EA had higher sensitivity than the HCL-33, which suggests that the HCL-33-EA, and carers more generally, could have better ability to correctly identify patients with hypomanic symptoms. The HCL-33 had higher specificity than the HCL-33-EA, which suggests that the HCL-33, and patients themselves, could have better ability in correctly identifying patients without hypomanic symptoms. Although the HCL-33 and the HCL-33-EA could be used according to different clinical purposes, joint use of the two scales is associated with more reliable assessment for $\mathrm{BD}$ patients.

The results showed a large difference in the optimal cut-off values between the HCL-33 and the HCL-33-EA, which could be partly due to the different perspective of the interviewers. For instance, a recent study found different feelings about clinical features of anxiety between patients and their carers using the pediatric short form (completed by patients) and proxy form (completed by carers) of the National Institutes of Health's Patient Reported Outcomes Measurement Information System scale. Carers tended to identify the existence of anxiety more than the patients themselves (33). In addition, recall bias may partly account for the discrepancy between mood disorder patients' and their carers' assessments (34). Compared to patients, their carers were less likely to have recall bias; therefore, the HCL33-EA version is more prone to identify BD than the HCL-33. The results showed there were significant, but poor agreement between the HCL-33 and the HCL-33-EA assessments $(k=0.36$, $p<0.001)$. The gap between the subjective and the external assessments indicates the importance of the combined use of the HCL-33 and the HCL-33-EA in identifying BD patients in clinical practice. Furthermore, the HCL-33-EA had a higher sensitivity, while the HCL-33 had a higher specificity in identifying BD patients from MDD patients in this study, which suggests that patients' carers were more likely to detect $\mathrm{BD}$, while patients themselves were more likely to recognize the absence of $\mathrm{BD}$. Hence, use of the two scales should be considered based on the assessment purpose in clinical research or daily practice (i.e., prefer sensitivity or specificity).

This study had several limitations. First, all participants were consecutively recruited in one major psychiatric hospital, and a relatively higher proportion of $\mathrm{BD}$ patients, therefore the findings cannot be generalized to patients in other clinical settings, which may bias the results to uncertain extent. However, the higher proportion of BD patients in the study sample reflects the real situation in daily practice in this major tertiary psychiatric hospital in China. Second, the ICD-10 is used in clinical practice in China, therefore, the diagnosis of BD-I and BD-II cannot be established. As such, we did not examine screening capacities for BD subtypes. In addition, the MiniInternational Neuropsychiatric Interview Version 5.0 cannot generate DSM-5 diagnoses. Therefore, certain specifiers related to $\mathrm{BD}$, such as mixed depression, could not be assessed. Third, we were unable to explore the differences in screening abilities between different carers who provided data on the HCL-33-EA (e.g., parents, spouses, or friends). Variation in the sources of self-reports should be examined in future studies. Fourth, the psychometric properties of the HCL-33-EA need to be tested with additional measures, such as the MDQ, as reference tools. Fifth, the possibility of recall bias could not be excluded, particularly in the HCL-33 assessment (34). Finally, diagnostic properties of screening instruments (e.g., sensitivity, specificity, PPV, and NPV) are associated with disease prevalence, study design, sampling method, and sample size (35). Similar to previous studies on the psychometric properties of the HCL scales (11, 2123 ), the sample size of this study was relatively small; therefore, 
our findings are tentative, and will need to be replicated in future studies with a larger sample size and a multicentre design.

\section{CONCLUSIONS}

In conclusion, both the HCL-33 and the HCL-33-EA appeared to have acceptable psychometric properties and screening abilities in accurately detecting and differentiating between $\mathrm{BD}$ and MDD. The two scales could facilitate identification of people with BD in clinical practice, and use of the two scales should be considered based on the assessment purpose in clinical research or daily practice (i.e., prefer sensitivity or specificity).

\section{DATA AVAILABILITY STATEMENT}

The Ethics Committee of Beijing Anding Hospital that approved the study prohibits the authors from making the research data set publicly available. Readers and all interested researchers may contact Dr. Gang Wang (Email address: gangwangdoc@gmail.com) for details. Dr. Wang could apply to the Ethics Committee of Beijing Anding Hospital for the release of the data.

\section{REFERENCES}

1. Phillips ML, Kupfer DJ. Bipolar disorder diagnosis: challenges and future directions. Lancet. (2013) 381:1663-71. doi: 10.1016/S0140-6736(13)60989-7

2. Chou CC, Lee IH, Yeh TL, Chen KC, Chen PS, Chen WT, et al. Comparison of the validity of the Chinese versions of the Hypomania Symptom Checklist32 (HCL-32) and Mood Disorder Questionnaire (MDQ) for the detection of bipolar disorder in medicated patients with major depressive disorder. Int $J$ Psychiatr Clin Pract. (2012) 16:132-7. doi: 10.3109/13651501.2011644563

3. Culpepper L. Misdiagnosis of bipolar depression in primary care practices. $J$ Clin Psychiatry. (2014) 75:e05. doi: 10.4088/JCP13019tx1c

4. Lish JD, Dimemeenan S, Whybrow PC, Price RA, Hirschfeld RMA. The National Depressive and Manic-Depressive Association (Dmda) survey of bipolar members. J Affect Disord. (1994) 31:281-94. doi: 10.1016/0165-0327(94)90104-X

5. Drancourt N, Etain B, Lajnef M, Henry C, Raust A, Cochet B, et al. Duration of untreated bipolar disorder: missed opportunities on the long road to optimal treatment. Acta Psychiatr Scand. (2013) 127:136-44. doi: 10.1111/j.1600-0447.2012.01917x

6. Mccombs JS, Ahn J, Tencer T, Shi L. The impact of unrecognized bipolar disorders among patients treated for depression with antidepressants in the fee-for-services California Medicaid (Medi-Cal) program: a 6-year retrospective analysis. J Affect Disord. (2007) 97:171-9. doi: $10.1016 /$ j.jad.2006.06018

7. Smith D, Ghaemi S, Craddock N. The broad clinical spectrum of bipolar disorder: implications for research and practice. J Psychopharmacol. (2008) 22:397-400. doi: 10.1177/0269881108089585

8. Angst J, Adolfsson R, Benazzi F, Gamma A, Hantouche E, Meyer TD, et al. The HCL-32: towards a self-assessment tool for hypomanic symptoms in outpatients. J Affect Disord. (2005) 88:217-33. doi: 10.1016/j.jad.2005.05011

9. Feng Y, Xiang YT, Huang W, Wang G, Feng L, Tian TF, et al. The 33-item Hypomania Checklist (HCL-33): a new self-completed screening instrument for bipolar disorder. J Affect Disord. (2016) 190:214-20. doi: 10.1016/j.jad.2015.09057

10. Lojko D, Dudek D, Angst J, Siwek M, Michalak M, Rybakowski JK. The 33item Hypomania Checklist (HCL-33) - a study of the consistency between

\section{ETHICS STATEMENT}

The studies involving human participants were reviewed and approved by Ethics Committee of Beijing Anding Hospital. The patients/participants provided their written informed consent to participate in this study. Written informed consent for publication was obtained.

\section{AUTHOR CONTRIBUTIONS}

Y-YW, GW, and Y-TX: study design. MF, CG, Y-YW, and YF: analysis and interpretation of data. Y-YW, GW, and Y-TX: drafting of the manuscript. $\mathrm{BH}$ and $\mathrm{GU}$ : critical revision of the manuscript. All authors approval of the final version for publication.

\section{FUNDING}

The study was supported by the University of Macau (MYRG2015-00230-FHS; MYRG2016-00005-FHS), the National Key Research \& Development Program of China (Grant No. 2016YFC1307200), the Beijing Municipal Science \& Technology Commission (Grant No. Z181100001718124), and the Beijing Talents Foundation (Grant No. 2017000021469G222).

self - and external assessments in Polish bipolar patients. Psychiatr Pol. (2016) 50:1085-92. doi: 10.12740/PP/66358

11. Bech P, Christensen EM, Vinberg M, Bech-Andersen G, Kessing LV. From items to syndromes in the Hypomania Checklist (HCL-32): psychometric validation and clinical validity analysis. J Affect Disord. (2011) 132:48-54. doi: 10.1016/j.jad.2011.01017

12. Wang YY, Feng Y, Xu DD, Huang W, Chow IHI, $\mathrm{Ng} \mathrm{CH}$, et al. The development and validation of a short version of the 33-item Hypomania Checklist (HCL-33). J Affect Disord. (2018) 235:206-10. doi: 10.1016/j.jad.2018.04019

13. Meyer TD, Hammelstein P, Nilsson LG, Skeppar P, Adolfsson R, Angst J. The Hypomania Checklist (HCL-32): its factorial structure and association to indices of impairment in German and Swedish nonclinical samples. Compr Psychiatry. (2007) 48:79-87. doi: 10.1016/j.comppsych.2006.07001

14. Yang HC, Yuan CM, Liu TB, Li LJ, Peng HJ, Liao CP, et al. Validity of the 32 item Hypomania Checklist (HCL-32) in a clinical sample with mood disorders in China. BMC Psychiatry. (2011) 11:84. doi: 10.1186/1471-244X-11-84

15. Poon Y, Chung KF, Tso KC, Chang CL, Tang D. The use of Mood Disorder Questionnaire, Hypomania Checklist-32 and clinical predictors for screening previously unrecognised bipolar disorder in a general psychiatric setting. Psychiatry Res. (2012) 195:111-7. doi: 10.1016/j.psychres.2011.07014

16. Yang HC, Xiang YT, Liu TB, Han R, Wang G, Hu C, et al. Hypomanic symptoms assessed by the HCL-32 in patients with major depressive disorder: a multicenter trial across China. J Affect Disord. (2012) 143:203-7. doi: 10.1016/j.jad.2012.06002

17. Gamma A, Angst J, Azorin JM, Bowden CL, Perugi G, Vieta E, et al. Transcultural validity of the Hypomania Checklist-32 (HCL-32) in patients with major depressive episodes. Bipolar Disord. (2013) 15:701-12. doi: 10.1111/bdi12101

18. Mosolov SN, Ushkalova AV, Kostukova EG, Shafarenko AA, Alfimov PV, Kostyukova $\mathrm{AB}$, et al. Validation of the Russian version of the Hypomania Checklist (HCL-32) for the detection of Bipolar II disorder in patients with a current diagnosis of recurrent depression. J Affect Disord. (2014) 155:90-5. doi: 10.1016/j.jad.2013.10029

19. Fang M, Wang YY, Feng Y, Ungvari GS, Ng CH, Wang G, et al. Exploration of the psychometric properties of the 33-item Hypomania Checklist - 
external assessment (HCL-33-EA). J Affect Disord. (2019) 245:987-90. doi: $10.1016 /$ j.jad.2018.11023

20. Zhang L, Cao XL, Wang SB, Zheng W, Ungvari GS, Ng CH, et al. The prevalence of bipolar disorder in China: a meta-analysis. J Affect Disord. (2017) 207:413-21. doi: 10.1016/j.jad.2016.08062

21. Vieta E, Sanchez-Moreno J, Bulbena A, Chamorro L, Ramos JL, Artal J, et al. Cross validation with the mood disorder questionnaire (MDQ) of an instrument for the detection of hypomania in Spanish: the 32 item hypomania symptom check list (HCL-32). J Affect Disord. (2007) 101:43-55. doi: 10.1016/j.jad.2006.09040

22. Wu YS, Angst J, Ou CS, Chen HC, Lu RB. Validation of the Chinese version of the hypomania checklist (HCL-32) as an instrument for detecting hypo (mania) in patients with mood disorders. J Affect Disord. (2008) 106:133-43. doi: $10.1016 / j . j a d .2007 .06004$

23. Mosolov S, Yaltonskaya P, Senko O, Angst J. Validation of the Russian version of the hypomania checklist (HCL-33) for the detection of bipolar disorder in patients with a current diagnosis of recurrent depression. J Affect Disord Rep. (2021) 4:100086. doi: 10.1016/j.jadr.2021100086

24. Sheehan DV, Lecrubier Y, Sheehan KH, Amorim P, Janavs J, Weiller E, et al. The Mini-International Neuropsychiatric Interview (M.I.N.I.): the development and validation of a structured diagnostic psychiatric interview for DSM-IV and ICD-10. J Clin Psychiatry. (1998) 59:22-33;quiz 34-57.

25. Si TM, Shu L, Dang WM, Su YA, Chen JX, Dong WT, et al. Evaluation of the reliability and validity of Chinese version of the mini international neuropsychiatric interview in patients with mental disorders (in Chinese). Chin Ment Health J. (2009) 23:493-503. doi: 10.3969/j.issn.1000-6729.2009.07.011

26. World Health Organization (1992). The ICD-10 Classification of Mental and Behavioural Disorders: Clinical Descriptions and Diagnostic Guidelines. Geneva: World Health Organization.

27. Faul F, Erdfelder E, Lang AG, Buchner A. G*Power 3: a flexible statistical power analysis program for the social, behavioral, biomedical sciences. Behav Res Methods. (2007) 39:175-91. doi: 10.3758/BF03193146

28. Hunsley J, Mash EJ. A Guide to Assessments that Work. Oxford: Oxford University Press (2008). doi: 10.1093/med:psych/9780195310641.0010001
29. Lee K, Oh H, Lee EH, Kim JH, Kim JH, Hong KS. Investigation of the clinical utility of the hypomania checklist 32 (HCL-32) for the screening of bipolar disorders in the non-clinical adult population. BMC Psychiatry. (2016) 16:124. doi: 10.1186/s12888-016-0831-8

30. Youden WJ. Index for rating diagnostic tests. Cancer. (1950) 3:32-5. doi: 10 . 1002/1097-0142(1950)3:1<32::AID-CNCR2820030106>3.0CO;2-3

31. Fleiss JL. Statistical Methods for Rates and Proportions. New York: Wiley (1981).

32. Byrt T, Bishop J, Carlin JB. Bias, prevalence and kappa. J Clin Epidemiol. (1993) 46:423-9. doi: 10.1016/0895-4356(93)9 0018-V

33. Gibson DJ, Glazier JJ, Olson KR. Evaluation of anxiety and depression in a community sample of transgender youth. JAMA Netw Open. (2021) 4:e214739. doi: 10.1001/jamanetworkopen.202 14739

34. Boschloo L, Nolen WA, Spijker AT, Hoencamp E, Kupka R, Penninx BW, et al. The Mood Disorder Questionnaire (MDQ) for detecting (hypo)manic episodes: its validity and impact of recall bias. J Affect Disord. (2013) 151:203-8. doi: 10.1016/j.jad.2013.05078

35. Steinberg DM, Fine J, Chappell R. Sample size for positive and negative predictive value in diagnostic research using case-control designs. Biostatistics. (2009) 10:94-105. doi: 10.1093/biostatistics/ kxn018

Conflict of Interest: The authors declare that the research was conducted in the absence of any commercial or financial relationships that could be construed as a potential conflict of interest.

Copyright @ 2021 Wang, Feng, Fang, Guo, Ungvari, Hall, Wang and Xiang. This is an open-access article distributed under the terms of the Creative Commons Attribution License (CC BY). The use, distribution or reproduction in other forums is permitted, provided the original author(s) and the copyright owner(s) are credited and that the original publication in this journal is cited, in accordance with accepted academic practice. No use, distribution or reproduction is permitted which does not comply with these terms. 